\title{
OTR6 Epidemiology of group a rotavirus diarrhea in the context of universal monovalent (G1P[8]) vaccination in Brazil
}

Eduardo de Mello Volotão ${ }^{1}$, Filipe Anibal Carvalho-Costa ${ }^{1}$, Mariela Martínez Gómez ${ }^{1}$, Marcelle Figueira Marques da Silva ${ }^{1}$, Tatiana Lundgren Rose ${ }^{1}$, Alexandre Madi Fialho', Rosane Maria Santos de Assis $^{1}$, José Paulo Gagliardi Leite ${ }^{1}$

${ }^{1}$ IOC, Fiocruz, Rio de Janeiro, RJ

Introduction: Group A rotavirus (RVA) vaccines have been considered strategic in order to reduce both mortality and hospitalization due to acute diarrheal diseases (AD), with other measures such as oral rehydration, breastfeeding, zinc administration and improvement of sanitation in developing countries. Brazil is extremely heterogeneous regarding income, living conditions, and access to basic sanitation.

Objective: To assess the changing epidemiology of $A D$ in Brazil, particularly RVA-AD, six years after the beginning (March 2006) of universal mass RVA monovalent (RV1: G1P[8] Rotarix®) vaccination.

Methodology: RVA was detected by enzyme immunoassay (EIA) and/or polyacrylamide gel electrophoresis (PAGE), and genotyped by multiplex semi-nested RT-PCR, as recommended by World Health Organization. Vaccine effectiveness (VE) was calculated as (1 - odds ratio of vaccination) X 100 (\%), stratified by age group and RVA genotype. Diarrhea-associated mortality data were gathered from the Brazilian Ministry of Health databank. Regression analyses were performed in order to estimate the annual expected number of diarrheaassociated deaths during the post-vaccination period based upon prevaccination annual trends.

Results: RVA detection decreased from 2010 (25.4\%) to 2012 (12.7\%). A reduction in the frequency of G2P[4]/G2P[NT] genotype was observed between 2010 (87\%) and 2012 (9.6\%). It was accompanied by an increase in detection of G3P[8]/G3P[NT] RVA genotype, $2.3 \%$ in $2010,39 \%$ in 2012 . VE against $A D$ requiring medical attention or hospitalization was higher in children aged 5-11 months (VE = $75 \%[54 \%-87 \%]$ ), declining in the older groups, being higher against P[8] RVA genotype (93\%[75\%-98\%]), when compared to G2P[4] (73\%[38\%-88\%]) RVA genotype. Diarrhea-specific mortality rates were higher in the Northeastern region, declining from $8.5 \%(1,971$ DD deaths [DD] / 23,275 total deaths [TD]) in 1996 to $2.4 \%$, (322 DD/13,197 TD) in 2011, when compared to the Southern region, where they decreased from $4.2 \%$ (387 DD/9,187 TD) in 1996 to $0.7 \%$ (30 DD/4,220 TD) in 2011. For children aged $\leq 1$ year old, the number of diarrhea deaths DT was below from expected in the year 2011 in regions Northeastern, Northern, Central-Western and Southern, and in 2009, 2010, and 2011 for the Northeastern region. 
Conclusion: RV1 has impacted substantially on the etiological profile of $A D$ by reducing significantly the burden of RVA disease. The number of deaths caused by AD was underestimated in the period of 2008-2011 in Brazil. A shift (G2/G3) in genotypes distribution has occurred between 2010 and 2012. RV1 vaccine, introduced in 2006, can be considered as one of other measures impacting AD burden in Brazil. Financial Support: PROEP/Fiocruz-CNPq, IOC-Fiocruz, PAPES VI Fiocruz-CNPq, CGLAB-SVS-MS, FAPERJ, OPAS.

Keywords: Rotavirus, Monovalent Vaccine, Epidemiology, Prevalence 